\title{
Regulatory T Cells with Effector Memory Phenotype and Glycaemic Control in Adult Type 1 Diabetes Mellitus
}

\author{
Elena Matteucci*, Luca Della Bartola and Ottavio Giampietro
}

Department of Clinical and Experimental Medicine, University of Pisa, Italy

\begin{abstract}
Background: There is controversy about absolute number, frequency, and status of homing markersof regulatory $\mathrm{T}$ (Treg) cells in patients with type 1 diabetes. We observed recently a considerable accumulation of terminally differentiated central memory subsets among $\mathrm{CD} 4^{+}$and $\mathrm{CD} 8^{+} \mathrm{T}$ cell expressing $\mathrm{CD} 26$ in patients with type 1 diabetes. The increased number of terminally differentiated central memory cells, which was positively associated with $\mathrm{HbA} 1 \mathrm{c}$ levels, could suggest life-long stimulation by protracted antigen exposure or a homeostatic defect in the regulation/contraction of immune responses.
\end{abstract}

Methods: We have analysed the phenotype of peripheral blood Treg cells in adult patients with type 1 diabetes using an 8-color flow cytometry assay panel for the characterisation of $C D 4^{+} C D 25^{\text {high }} \mathrm{CD} 127^{-}$Treg cells into naïve $\left(\mathrm{N}, \mathrm{CCR} 7^{+} \mathrm{CD} 45 \mathrm{RA}^{+}\right)$, central memory $\left(\mathrm{CM}, \mathrm{CCR} 7^{+} \mathrm{CD} 45 \mathrm{RA} \mathrm{A}^{-}\right.$), and effector memory (EM, CCR7-CD45RA') cells. We also examined the expression of two additional markers: the serine protease CD26 that has been recently suggested as a negative selection marker for human Treg cells, and the cutaneous lymphocyte-associated antigen, given that the bidirectional homing of Treg cells between the skin and lymph nodes is important for efficient regulation.

Results: We found that patients with type 1 diabetes had normal Treg frequencies but a low proportion of CCR7 EM Treg cells, which was inversely correlated with both a long-term indicator of glycaemic control such as $\mathrm{HbA} 1 \mathrm{c}$ and an indicator of renal function such as plasma creatinine.

Conclusion: In our opinion, present results seem to support the second of the two initial hypotheses, i.e. a defect in regulation/suppression of immune homeostasis in diabetic people. Moreover, the worse glycaemic control (evaluated by $\mathrm{HbA} 1 \mathrm{c}$ ), the higher frequency of immune defects and more severe.

Keywords: Type 1 diabetes mellitus; Regulatory $\mathrm{T}$ cells; Effector memory; CD45RA; CCR7; HbA1c

\section{Introduction}

In autoimmune type 1 diabetes, innate and adaptive immune mechanisms progressively destroy insulin-secreting $\beta$ cells in the islets of the pancreas [1]. To date, islet cell antibodies and antibodies directed against proteins found in $\beta$ cells are the best known markers of type 1 diabetes in humans [2]. Our knowledge of diabetes development as a T-cell-mediated autoimmune disease derives primarily from animal models [3]. Recent studies in humans provide evidence for an altered immune balance between the expansion of proinflammatory Th17 cells and defects in regulatory $\mathrm{T}$ (Treg) cell-mediated suppression [4,5]. Nevertheless, human data interpretation is problematic because 1) T cells are always in adynamic state of differentiation, 2) literature shows a wide range in terms of patients' age and disease duration, 3) chronological changes of $\mathrm{T}$ cell subsets that occur in recent onset and long lasting diabetes are unknown, but the modulatory effect of insulin on T cell receptor mediated calcium signalling has been found blunted in long-term type 1 diabetes [6], 4) finally, metabolism is a fundamental determinant of $\mathrm{T}$ cell fate and redox-metabolic remodelling regulate immune responses by Treg cells [7,8]. Initial reports on impaired frequency or function of Treg cells in type 1 diabetes have not been confirmed, and to date there is controversy or little information about their absolute number, frequency, and status of homing markers [5]. The present study was designed to analyse the phenotype of peripheral blood Treg cells in adult patients with type 1 diabetes using an 8-colour flow cytometry assay panel for the characterisation of $\mathrm{CD}^{+} \mathrm{CD} 25^{\text {high }} \mathrm{CD} 127^{-}$Treg cells into naïve $\left(\mathrm{N}, \mathrm{CCR} 7^{+} \mathrm{CD} 45 \mathrm{RA}^{+}\right)$, central memory $\left(\mathrm{CM}, \mathrm{CCR}^{+} \mathrm{CD} 45 \mathrm{RA}\right)$, and effector memory (EM, CCR7 CD45RA') cells. We also examined the expression of two additional markers: 1 ) the serine protease CD26 that has been recently suggested as a negative selection marker for human Treg cells [9], and 2) the cutaneous lymphocyte-associated antigen (CLA), given that the bidirectional homing of Treg cells between the skin and lymph nodes is important for efficient regulation [10] and skin of people with type 1 diabetes is constantly exposed to external micro-insults (repeated daily subcutaneous injections).

\section{Materials and Methods}

Fresh peripheral blood samples were obtained from 30 patients with type 1 diabetes (diabetes duration $25 \pm 13$ years, range 4-53 years; daily insulin dose $0.57 \pm 0.18 \mathrm{IU} / \mathrm{kg}$ ) and 20 age- and sexmatched healthy non-diabetic control subjects with no family history of the disease whose clinical characteristics are shown in table 1 . The diagnosis of type 1 diabetes was confirmed by the presence of detectable autoantibodies, fasting C peptide $<0.4 \mathrm{ng} / \mathrm{mL}$, and insulin monotherapy since diagnosis; no patient received medical treatment except insulin, antihypertensive (10 subjects) and/or lipid-lowering ( 9 subjects) drugs. None of the recruited control subjects had clinical evidence of illness

${ }^{*}$ Corresponding author: Dr. Elena Matteucci, Dipartimento di Medicina Clinica Sperimentale, Via Roma 6756126 Pisa, Italy, Tel: 0039050 993246; Fax: 0039 050 993520; E-mail: elena.matteucci@med.unipi.it

Received April 05, 2013; Accepted May 02, 2013; Published May 08, 2013

Citation: Matteucci E, Bartola LD, Giampietro O (2013) Regulatory T Cells with Effector Memory Phenotype and Glycaemic Control in Adult Type 1 Diabetes Mellitus. J Diabetes Metab S12: 003. doi:10.4172/2155-6156.S12-003

Copyright: (c) 2013 Matteucci E, et al. This is an open-access article distributed under the terms of the Creative Commons Attribution License, which permits unrestricted use, distribution, and reproduction in any medium, provided the original author and source are credited. 


\begin{tabular}{|c|c|c|c|}
\hline Parameter & Type 1 diabetes & Control subjects & $\mathbf{P}$ \\
\hline F/M & $18 / 12$ & $11 / 9$ & $<0.5$ \\
\hline Age $($ years $)$ & $48 \pm 11$ & $45 \pm 13$ & 0.444 \\
\hline BMI $\left(\mathrm{kg} / \mathrm{m}^{2}\right)$ & $25 \pm 3$ & $24 \pm 4$ & 0.2105 \\
\hline MBP $(\mathrm{mmHg})$ & $91 \pm 11$ & $92 \pm 11$ & 0.5775 \\
\hline FPG $(\mathrm{mg} / \mathrm{dL})$ & $167 \pm 63$ & $90 \pm 10$ & $<0.0001$ \\
\hline HbA1c $(\mathrm{mmol} / \mathrm{mol})$ & $67 \pm 9$ & $38 \pm 2$ & $<0.0001$ \\
\hline Fructosamine $(\mu \mathrm{mol} / \mathrm{L})$ & $349 \pm 36$ & $231 \pm 15$ & $<0.0001$ \\
\hline Creatinine $(\mathrm{mg} / \mathrm{dL})$ & $0.85 \pm 0.14$ & $0.86 \pm 0.20$ & 0.464 \\
\hline Neutrophils $(/ \mu \mathrm{L})$ & $4024 \pm 1521$ & $3646 \pm 733$ & 0.307 \\
\hline Lymphocytes $(/ \mu \mathrm{L})$ & $2308 \pm 754$ & $1952 \pm 420$ & 0.061 \\
\hline T CD4+ $(\%)$ & $47.0[14.5]$ & $47.0[10.0]$ & 0.6920 \\
\hline Treg $(\%)$ & $5.4[2.3]$ & $4.9[1.7]$ & 0.4759 \\
\hline CM Treg $(\%)$ & $52.8[16.3]$ & $44.2[17.4]$ & 0.0129 \\
\hline Naïve Treg $(\%)$ & $15.9[12.5]$ & $12.9[9.8]$ & 0.2347 \\
\hline EM Treg $(\%)$ & $27.8[9.1]$ & $37.6[16.4]$ & 0.0014 \\
\hline
\end{tabular}

MB: Mean Blood Pressure; FPG: Fasting Plasma Glucose; Treg: regulatory T cells; CM: Central Memory; EM: Effector Memory; Data are mean \pm SD or median [interquartile range]

Table 1: Clinical, laboratory, andimmunophenotypic characteristics of study participants, control subjects and patients with type 1 diabetes.

or was taking any drugs. All subjects gave informed consent according to institutional guidelines. The medical examination included medical history and physical exam. Body Mass Index (BMI) was calculated as $\mathrm{kg} / \mathrm{m}^{2}$. Mean blood pressure was calculated as diastolic blood pressure $+33 \%$ of pulse pressure. Blood chemistry analysis included: complete blood count, Fasting Plasma Glucose (FPG), fructosamine, HbAlc, plasma lipids, creatinine, hepatic enzymes, bilirubin, urinary albuminto-creatinine ratio.

Eight-colour immunophenotypic analyses were performed on peripheral whole blood lymphocytes by the standard direct immunofluorescence technique using flow cytometry (FACSCanto II, BD Biosciences, Milan, Italy) with a panel of monoclonal antibodies (mAb, Becton Dickinson Immunocytometry Systems, San Jose, California, USA). The following antibody clones were used: allophycocyanin (APC)-Cy7-conjugated mAb against CD45 (2D1), Horizon V500-conjugated $\mathrm{mAb}$ against CD4 (RPA-T4), APCconjugated $\mathrm{mAb}$ against $\mathrm{CD} 25$ (2A03), APC-conjugated $\mathrm{mAb}$ against CD27 (L128), Horizon V450-conjugated mAb against CD197 (CCR7, 150503), phycoerythrin (PE)-conjugated $\mathrm{mAb}$ against CD45RA (HI100), PE-conjugated mAb against CD26 (M-A261), Fluorescein Isothiocyanate (FITC)-conjugated $\mathrm{mAb}$ against CLA (HECA452). A minimum of 100,000 events for sample was acquired. Postacquisition analysis was performed using FlowJo software (Tree Star Inc). A representative gating strategy for Treg immuniphenotyping by flow cytometry is shown in figure 1. Briefly, lymphocytes were gated according to their CD45 intensity and side-scatter characteristics. Then a gate was placed around the $\mathrm{CD} 4^{+}$population followed by a gate on the $\mathrm{CD} 25^{+} \mathrm{CD} 127^{\mathrm{lo} /}$ population, and then gates were applied for sorting $\mathrm{CD}_{45 \mathrm{RA}^{+}}$and CD45RA populations. Finally, the sorted cells were analysed to assess the intensity of CD26 and CLA intensity.

Statistical analysis was performed using Aabel 3 (Gigawiz, Oklahoma City, Oklahoma, USA). Data were expressed as mean \pm SD (or median and interquartile range for not normally distributed lymphocyte subpopulation counts). The unpaired Student's $t$ test or Mann-Whitney U-test were used to compare two independent samples. The Spearman rank correlation test was used for the correlation exploration of two variables. Multiple regression analysis was used to determine independent factors. A $p$ value of less than 0.05 was considered statistically significant.

\section{Results}

Clinical and laboratory characteristics of study participants are reported in table 1. Comparing total lymphocyte counts as well as the percentages of peripheral $\mathrm{CD} 4^{+} \mathrm{T}$ cells and Treg cells of control and diabetic subjects, no significant difference was found between groups (Table 1). Notwithstanding the lymphocyte counts were similar between groups, immunophenotypic analyses of peripheral Treg subsetsfrom adults with type 1 diabetes evidenced an increase in percentages of Treg cells with a CM phenotype $(\mathrm{p}<0.05)$, whereas EM phenotype cells were significantly decreased $(p<0.01)$ (Table 1$)$. The frequency of naïve Treg percent did not differ significantly between diabetic and control subjects (Table 1). The percentages of Treg cells with an EM phenotype correlated negatively with HbA1c levels (Spearman's rank correlation coefficient $\mathrm{rho}=-0.41, \mathrm{p}=0.0042$ ), and plasma creatinine values $(\mathrm{rho}=-0.28, \mathrm{p}=0.023)$. Multivariate regression analysis was used to identify determinants of the peripheral EM Treg cells in the whole study population. The following variables were confirmed by backward stepwise multiple regression $(\mathrm{r}=0.55, \mathrm{p}=0.0002)$ : HbAlc (beta coefficient -0.321 , standard error $0.086, p=0.0005$ ) and creatinine $(-22.16,8.53, \mathrm{p}=0.0125)$. There was no association between diabetes duration and differences in the proportions of Treg subsets.

The expression of CLA (Mean Fluorescence Intensity, MFI), associated with a CM or EM phenotype was similar between the two study groups: median 79.9 [IQR 39.6] in CM Treg cells of the control subjects vs. 64.7 [56.8] in those of patients with type 1 diabetes $(\mathrm{p}=0.205) ; 78.2$ [35.7] in EM Treg cells of the control subjects vs. 64.3 [59.7] in those of patients with type 1 diabetes $(p=0.140)$. Treg subsets of control subjects and patients with type 1 diabetes displayed similar expression of CD26: 132.0 [60.5] in CM Treg cells of the control subjects vs. 140.0 [105.0] in those of patients with type 1 diabetes $(\mathrm{p}=0.327) ; 58.3$ [11.4] in EM Treg cells of the control subjects vs. 65.5 [40.7] in those of patients with type 1 diabetes $(\mathrm{p}=0.422)$.

\section{Discussion}

We observed recently a considerable accumulation of terminally differentiated central memory subsets among $\mathrm{CD} 4^{+}$and $\mathrm{CD}^{+} \mathrm{T}$ cell expressing CD26 in patients with type 1 diabetes that could suggest life-long stimulation by protracted antigen exposure or a homeostatic defect in the regulation/contraction of immune responses [11]. For this reason, we have analysed the frequency and phenotype of Treg cells using a combination of phenotypic markers associated with immune suppression (CD25 $5^{\text {high }}$, CD127), cell differentiation (CCR7, CD45RA), cell activation/memory for $\mathrm{T}$ lymphocytes but possible negative selection marker for Treg cells (CD26), and the skin-homing CLA. In the present study, we found that patients with type 1 diabetes and normal Treg counts had a low proportion of CCR7- EM Treg cells, which was inversely correlated with both a long-term indicator of glycaemic control such as $\mathrm{HbAlc}$ and an indicator of renal function such as plasma creatinine.

There is no previous study that has analysed circulating Treg cells with CM/EM phenotypes in adult humans with type 1 diabetes with which we can compare our data. The analysis of Treg cells in humans is rather complicated. Treg cells are characterised as cells that are $\mathrm{CD}_{4}^{+}$, express constitutively high levels of CD25, and lack the expression of CD27, whose levels inversely correlate with FoxP3 expression. The transcription factor FoxP3 is a specific Treg marker, but is intracellular and shows an activation-dependent expression in $\mathrm{CD} 4^{+} \mathrm{CD} 25^{-}$effector $\mathrm{T}$ cells [5]. Moreover, conventional/effector $\mathrm{T}$ cells express CD25 upon TCR-mediated activation. Additionally, CD26 has recently 

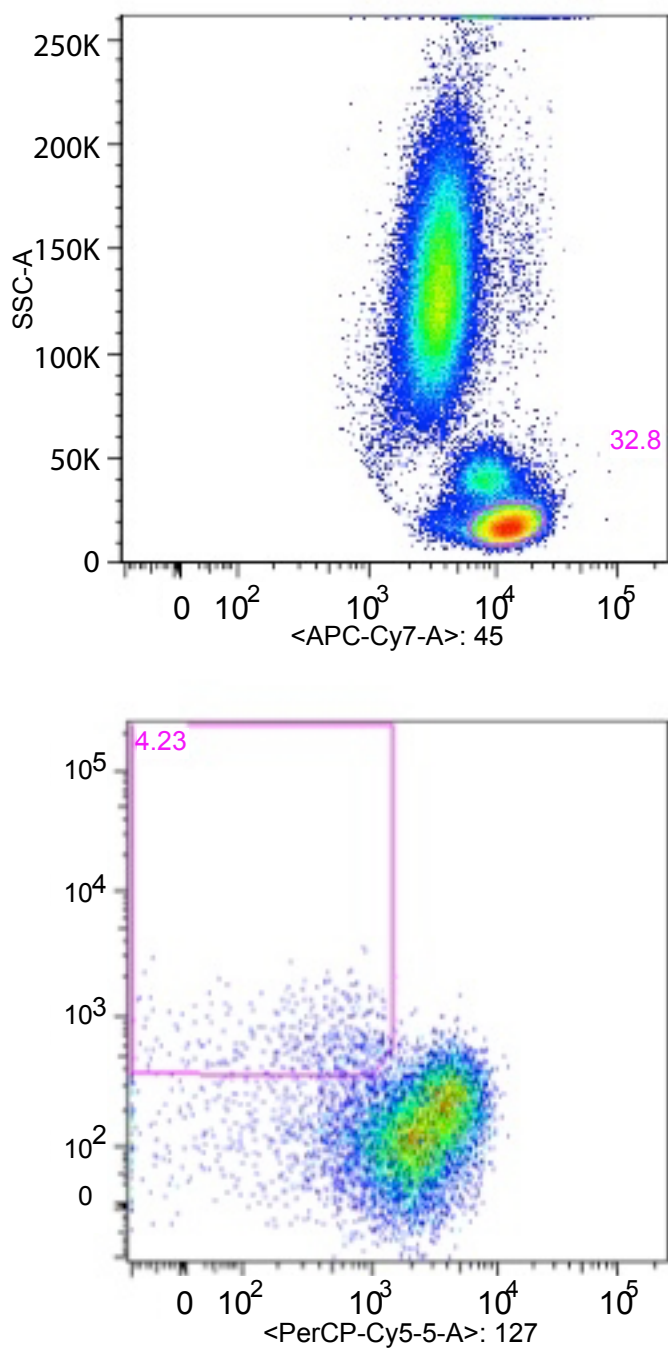
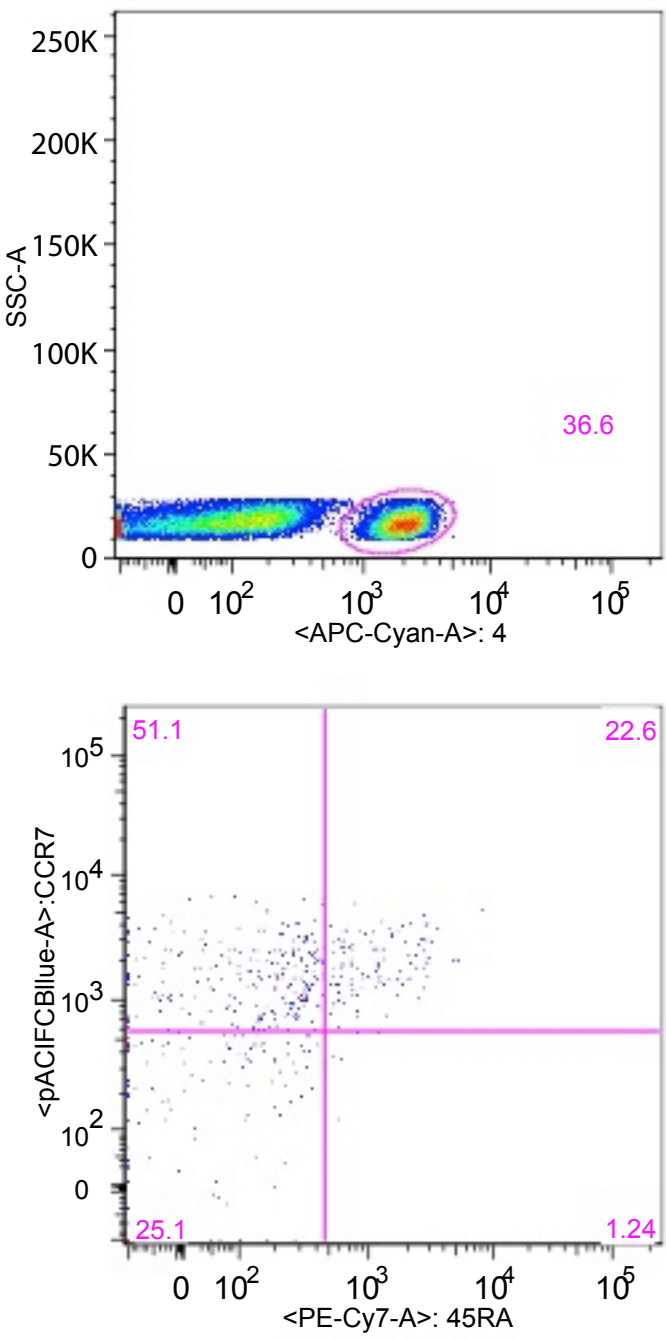

Figure 1: Representative plots showing the gating strategies for $C D 4^{+} C D 25^{\text {high }} C D 127^{-}$regulatory $T$ cells $\left(\right.$ Tregs) by $C C R 7^{+} C D 45 R A^{+}($naïve, $N), C C R 7^{+} C D 45 R A-$ (central memory, CM), and CCR7-CD45RA- (effector memory EM) phenotypes.

been suggested as a useful negative selection marker for human Treg cells [9]. Regulatory $\mathrm{T}$ cells play a central role in maintenance of self-tolerance and immune homeostasis. Like conventional CD4 ${ }^{+}$ effector T cells, Treg cells are a heterogeneous population that is not at a terminal stage of differentiation but displays a significant degree of plasticity. Treg cells can be differentiated into phenotypically (and functionally) distinct subsets by acquiring distinct patterns of transcription factor and chemokine receptor expression; additionally, they may modify their phenotypes in response to environmental cues $[12,13]$. The fraction of circulating Treg cells, which harbours a naivelike phenotype, is considered to preferentially migrate into secondary lymphoid tissues and act predominantly in controlling the priming phase of immune responses occurring in these sites. Upon antigen stimulation, naive $\mathrm{T}$ cells get activated and acquire a new profile of tissue-specific homing receptors guiding them to peripheral tissues drained by the lymph node $[14,15]$. Hence, the heterogeneity in tissue-specific homing patterns of effector/memory Treg cells enables their efficient migration into peripheral tissues and inflamed sites where Treg-mediated suppression plays a central role in the control of on-going inflammatory responses [14]. The CM-like population is considered to control $\mathrm{T}$ cell homeostasis and the onset of immune responses in lymphoid organs, whereas the EM-like population controls the effector phase of immune responses in peripheral tissues [16]. Furthermore, not only the mechanisms underlying tissue-specific lymphocyte migration, but also the molecular control of the exit phase is critical in regulating the suppressive activity of effector/memory Treg cells. Effector/memory Treg cells use the CCR7 pathway to exit from peripheral tissues so that Treg lacking functional CCR7 may accumulate in peripheral tissues [14]. Consequently, according to the hypothesis of Menning et al. [14], the lower percentages of circulating EM Treg cells associated with poor glycaemic control could suggest that cells exposed to chronic hyperglycaemia remain trapped in peripheral tissues (since CCR7 deficiency does not supports their emigration) where they might result in more efficient suppression. The majority of studies regarding Treg migration to the periphery are in the contest of inflammation, infection, or cancer; they do not address Treg migration in normal conditions as assumed here on the basis of history, clinical examination, and normal laboratory tests including white blood cells 
and neutrophils. However, hyperglycaemia-mediated dysregulation of Treg numbers and their ratio to conventional effector cells could lead to changes in immune suppression and homeostasis, as it does elsewhere [17]. Indeed, although reports suggest an increased susceptibility of diabetic people to infections, little information is available on possible underlying immunologic defects. Besides the direct connection between metabolism and differentiation [7,8], the altered Treg phenotype seen in diabetic people - correlated with indices of glycaemic control (HbAlc) and renal function (creatinine) - could be interpreted taking into account the role of protein glycosylation and glycoxidation on molecular physicochemical properties. Upon recognition of antigen, Treg cells lose their naïve phenotype and become effector- or memorylike cells expressing distinct surface markers. There is evidence that $\mathrm{T}$ Cell Receptor (TCR) recognition and TCR signalling have a key role in influencing the phenotype, function, and localisation of Treg cells in vivo [18]. In this regard, it is noteworthy that the functional avidity of a $\mathrm{T}$ cell, as reflected by responsiveness to antigen, is modulated by $\mathrm{T}$ cell surface $\mathrm{O}$ - or $\mathrm{N}$-glycosylation, with increased glycosylation of $\mathrm{T}$ cell surface proteins associated with an increased activation threshold. The reduced $\mathrm{N}$-glycosylation of the TCRs appeared to specifically result in increased of TCR mobility at the cell surface, enhanced TCR clustering, improved TCR engagement/down-modulation, and, consequently, increased functional avidity of the T cell [19]. In addition to the signalling via TCR, costimulatory signals are in turn required for the control of Treg development and homeostasis [20]. Although the physiological significance of CD28 glycosylation is at present unknown, $\mathrm{N}$-glycosylation negatively regulates CD28-mediated $\mathrm{T}$ cell adhesion and costimulation [21]. Advanced Glycation End products (AGE) include a variety of protein adducts whose accumulation has been implicated in tissue damage associated with diabetes, renal failure, and aging. Among AGE, glycoxidation products, such as carboxymethyllysine, pentosidine, and malondialdehyde-lysine, are all formed under oxidative stress by carbonyl amine chemistry between protein amino group and carbonyl compounds derived from autoxidation of carbohydrates and lipids. Recent evidence suggests that carbonylation of the cystoskeletal protein actin leads to aggregate formation contributing to T cellular function impairment [22].

Unlike findings obtained in Type 1 diabetes, $\mathrm{Bi}$ et al. found that untreated HIV type 1 -infected patients with low $\mathrm{CD} 4^{+}$counts had high frequency of CCR7-CD45RA- Treg. Cell proliferation was higher in Treg than in conventional $\mathrm{T}$ cells, the proliferation of Treg correlated positively with the proportion of CCR7CD45RA- Treg, and HIV viral load showed a positive correlation with both Treg proliferation and the proportion of CCR7-CD45RA- Treg [23]. Reverting the reasoning of $\mathrm{Bi}$ et al. [23], poorly controlled type 1 diabetes seems to be associated with a decreased proportion of CCR7 CD45RA among Treg cells in the peripheral blood, as if hyperglycaemia could inactivate the differentiation of Treg as well as inhibit CCR7-CD45RA- Treg migration to non-lymphoid tissues. Unfortunately, in this preliminary clinical study, we have neither stained $\mathrm{CD} 4^{+}$cells for the proliferation markers, nor evaluated the suppressive activity of CCR $7^{+} \mathrm{CD} 45 \mathrm{RA}-$ and CCR7-CD45RA-Treg.

\section{Conclusion}

In our previous study, $\mathrm{CD} 4^{+}$and $\mathrm{CD} 8^{+} \mathrm{T}$ cells expressing $\mathrm{CD} 26^{+}$ showed a distinctive differentiation profile: 1) percentages and absolute numbers of $\mathrm{CM}$ and naive cells were reduced, whereas those of terminally differentiated effector memory cells were markedly increased, and 2) the indices of intermediate- and long-term glycaemic control were associated negatively with the number of $\mathrm{CM}$ and naive cells while positively with the number of TEMRA cells. At the time, we hypothesised that the considerable accumulation of terminally differentiated effector memory cells T cells in our patients suggests lifelong stimulation by protracted antigen exposure (viruses, other agents, or residual self-antigens?) or a homeostatic defect in the regulation/ contraction of immune responses. In our opinion, present results seem to support the second hypothesis and confirm that the worse glycaemic control, the higher frequency of immune defects and more severe. Since modulation of CCR7 expression and function on Treg cells can be expected to result in contrasting effects on immune reactions in the context of diabetes, greater understanding of the mechanisms through which immunity is influenced by the metabolic milieu could shed light to the immunopathology of diabetes and lead to improved treatment.

\section{Acknowledgement}

We recognize and appreciate the technical expertise of Massimo Ghimenti and Stefano Giuntini, Department of Clinical and Experimental Medicine, Division of Hematology, University of Pisa.

\section{References}

1. Harrison LC, Honeyman MC, Morahan G, Wentworth JM, Elkassaby S, et al (2008) Type 1 diabetes: lessons for other autoimmune diseases? J Autoimmun 31: 306-310.

2. Winter WE, Schatz DA (2011) Autoimmune markers in diabetes. Clin Chem 57: $168-175$

3. Driver JP, Serreze DV, Chen YG (2011) Mouse models for the study of autoimmune type 1 diabetes: a NOD to similarities and differences to human disease. Semin Immunopathol 33: 67-87.

4. Shao S, He F, Yang Y, Yuan G, Zhang M, et al. (2012) Th17 cells in type 1 diabetes. Cell Immunol 280: 16-21.

5. Zhang Y, Bandala-Sanchez E, Harrison LC (2012) Revisiting regulatory T cells in type 1 diabetes. Curr Opin Endocrinol Diabetes Obes 19: 271-278.

6. Demkow U, Winklewski P, Ciepiela O, Popko K, Lipinska A, et al. (2012) Modulatory effect of insulin on $T$ cell receptor mediated calcium signaling is blunted in long lasting type 1 diabetes mellitus. Pharmacol Rep 64: 150-156.

7. Michalek RD, Gerriets VA, Jacobs SR, Macintyre AN, Maclver NJ, et al. (2011) Cutting edge: distinct glycolytic and lipid oxidative metabolic programs are essential for effector and regulatory CD4+ T cell subsets. J Immunol 186: 3299 3303.

8. Rubartelli A, Sitia R (2009) Chemo-metabolic regulation of immune responses by Tregs. Nat Chem Biol 5: 709-710.

9. Salgado FJ, Pérez-Díaz A, Villanueva NM, Lamas O, Arias P, et al. (2012 CD26: a negative selection marker for human Treg cells. Cytometry A 81: 843 855

10. Matsushima $\mathrm{H}$, Takashima $\mathrm{A}$ (2010) Bidirectional homing of Tregs between the skin and lymph nodes. J Clin Invest 120: 653-656.

11. Matteucci E, Ghimenti M, Di Beo S, Giampietro O (2011) Altered proportions of naïve, central memory and terminally differentiated central memory subsets among CD4+ and CD8 + T cells expressing CD26 in patients with type 1 diabetes. J Clin Immunol 31: 977-984

12. Tosello V, Odunsi K, Souleimanian NE, Lele S, Shrikant $P$, et al. (2008) Differential expression of CCR7 defines two distinct subsets of human memory CD4+CD25+ Tregs. Clin Immunol 126: 291-302.

13. Duhen T, Duhen R, Lanzavecchia A, Sallusto F, Campbell DJ (2012) Functionally distinct subsets of human FOXP3+ Treg cells that phenotypically mirror effector Th cells. Blood 119: 4430-4440.

14. Menning A, Höpken UE, Siegmund K, Lipp M, Hamann A, et al. (2007) Distinctive role of CCR7 in migration and functional activity of naive- and effector/memory-like Treg subsets. Eur J Immunol 37: 1575-1583.

15. Li L, Boussiotis VA (2011) Molecular and functional heterogeneity of $T$ regulatory cells. Clin Immunol 141: 244-252.

16. Grindebacke H, Stenstad H, Quiding-Järbrink M, Waldenström J, Adlerberth I, 
Citation: Matteucci E, Bartola LD, Giampietro O (2013) Regulatory T Cells with Effector Memory Phenotype and Glycaemic Control in Adult Type 1 Diabetes Mellitus. J Diabetes Metab S12: 003. doi:10.4172/2155-6156.S12-003

et al. (2009) Dynamic development of homing receptor expression and memory cell differentiation of infant CD4+CD25high regulatory T cells. J Immunol 183: 4360-4370.

17. Mailloux AW, Young MR (2010) Regulatory T-cell trafficking: from thymic development to tumor-induced immune suppression. Crit Rev Immunol 30: 435-447.

18. Li L, Boussiotis VA (2011) Molecular and functional heterogeneity of $T$ regulatory cells. Clin Immunol 141: 244-252.

19. Kuball J, Hauptrock B, Malina V, Antunes E, Voss RH, et al. (2009) Increasing functional avidity of TCR-redirected T cells by removing defined $\mathrm{N}$-glycosylation sites in the TCR constant domain. J Exp Med 206: 463-475.
20. Ohkura N, Sakaguchi S (2010) Regulatory T cells: roles of T cell receptor for their development and function. Semin Immunopathol 32: 95-106.

21. Ma BY, Mikolajczak SA, Yoshida T, Yoshida R, Kelvin DJ, et al. (2004) CD28 $T$ cell costimulatory receptor function is negatively regulated by $\mathrm{N}$-linked carbohydrates. Biochem Biophys Res Commun 317: 60-67.

22. Castro JP, Ott C, Jung T, Grune T, Almeida H (2012) Carbonylation of the cytoskeletal protein actin leads to aggregate formation. Free Radic Biol Med 53: 916-925.

23. Bi X, Suzuki $Y$, Gatanaga $H$, Oka S (2009) High frequency and proliferation of CD4+ FOXP3+ Treg in HIV-1-infected patients with low CD4 counts. Eur $J$ Immunol 39: 301-309. 